\title{
O perfil dos cirurgiões de catarata no sul do Brasil
}

\author{
The profileof cataract surgeons in southern Brazil
}

\author{
Rafael Faustini Loeff ${ }^{1}$ \\ Zélia Maria da Silva Corrêa ${ }^{2}$ \\ Alexandre Seminoti Marcon ${ }^{3}$ \\ André Moraes Freitas ${ }^{4}$ \\ Ítalo Mundialino Marcon ${ }^{5}$
}

Estudo realizado no Departamento de Oftalmologia da Irmandade da Santa Casa de Misericórdia de Porto Alegre (ISCMPA).

Médico oftalmologista, Setor de Córnea e Doenças Externas do Departamento de Oftalmologia da Irmandade da Santa Casa de Misericórdia de Porto Alegre (ISCMPA) e Fundação Faculdade Federal de Ciências Médicas de Porto Alegre (FFFCMPA).

${ }^{2}$ Médica oftalmologista, Departamento de Oftalmologia da Irmandade da Santa Casa de Misericórdia de Porto Alegre (ISCMPA) e Fundação Faculdade Federal de Ciências Médicas de Porto Alegre (FFFCMPA). Doutora em Medicina-Oftalmologia, Universidade Federal de São Paulo (UNIFESP).

${ }^{3}$ Médico oftalmologista, Setor de Córnea e Doenças Externas do Departamento de Oftalmologia da Irmandade da Santa Casa de Misericórdia de Porto Alegre (ISCMPA)e Fundação Faculdade Federal de Ciências Médicas de Porto Alegre (FFFCMPA). Pós-graduando, nível doutorado pela Universidade de São Paulo - (USP).

${ }^{4}$ Médico oftalmologista, Departamento de Oftalmologia da Irmandade da Santa Casa de Misericórdia de Porto Alegre (ISCMPA) e Fundação Faculdade Federal de Ciências Médicas de Porto Alegre (FFFCMPA).

${ }_{5}^{5}$ Médico Chefe do Departamento de Oftalmologia da Irmandade da Santa Casa de Misericórdia de Porto Alegre (ISCMPA) e Fundação Faculdade Federal de Ciências Médicas de Porto Alegre (FFFCMPA). Mestre e Doutor em Medicina - Oftalmologia pela Universidade Federal de São Paulo (UNIFESP). Professor Livredocente da Disciplina de Oftalmologia, Departamento de Oftalmo-Otorrino Fundação Faculdade Federal de Ciências Médicas de Porto Alegre (FFFCMPA).

Endereço para correspondência: Zélia M. S. Corrêa Av. Nilo Peçanha, 2421 - Porto Alegre (RS) CEP 91330-001 E-mail: zmcorrea@terra.com.br

Nota Editorial: Pela análise deste trabalho e por sua anuência na divulgação desta nota, agradecemos ao Dr. Paiva Gonçalves Neto.

Recebido para publicação em 01.08.2002 Aceito para publicação em 07.04.2003

\begin{tabular}{|l|}
\hline RESUMO \\
\hline Objetivos: Avaliar o perfil e as preferências dos cirurgiões de catarata que \\
atuam na região sul do Brasil. Métodos: Um questionário sobre a rotina da \\
cirurgia de catarata foi elaborado pelos autores e enviadoa 1.000 oftalmologis- \\
tas dos três estados que compõem essa região do país (Paraná, Santa \\
Catarina e Rio Grande do Sul). Ao questionário foi anexada uma carta \\
explicando os propósitos do trabalho, e um envelope pré-selado. A avalia- \\
ção dos dados colhidos foi realizada por meio do pacote estatístico SPSS 8.0 \\
for Windows ${ }^{\circledR}$. Resultados: Apenas 210 questionários (21\%) foram re- \\
metidos pelos entrevistados, devidamente preenchidos. Pela análise dos \\
itens nele contidos, observou-se que fazem parte das preferências desses \\
cirurgiões: o uso da anestesia peribulbar e a extração da catarata pela técnica \\
extracapsular, com implantação de lentes de 5 e 6 milímetros de área óptica \\
e sutura por meio de pontos separados. A facoemulsificação só é usada por \\
39\% desses cirurgiões, em cerca de $60 \%$ das suas operações. Conclusão: \\
Apesar dos constantes avanços tecnológicos observados nessa área da \\
medicina, viu-se que técnicas modernas, como a facoemulsificação, não são \\
aindautilizadas rotineiramente pelos oftalmologistas da região sul do Brasil \\
que participaram da presente pesquisa.
\end{tabular}

Descritores: Catarata; Facoemulsificação; Extração de catarata; Área de atuação profissional; Médicos; Conduta na prática dos médicos/estatística \& dados numéricos

\section{INTRODUÇÃOO}

A oftalmologia é, provavelmente, uma das especialidades médicas mais beneficiadas pelos avanços tecnológicos ocorridos nas últimas décadas e a cirurgia de catarata pode ser tomada como um bom exemplo disso ${ }^{(1)}$. O aperfeiçoamento da técnica operatória, aliado à sofisticação dos equipamentos e das lentes intra-oculares, tornou o procedimento mais rápido, seguro e preciso. Hoje é possível que o paciente recupere sua acuidade visual em um curto espaço de tempo, sem a ajuda de correção óptica para longe ${ }^{(1-2)}$.

Ao que parece, entretanto, nem todos os portadores de catarata do país têm sido beneficiados por esses avanços. Por isso, procurou-se elaborar um estudo que avaliasse a proporção com que tais recursos estão de fato disponíveis, pesquisando a conduta dos cirurgiões de catarata de uma determinada região do país. Embora limitado a uma área geográfica, tal estudo pode servir para ter-se uma noção do que vem acontecendo no restante do país.

\section{OBJETIVOS}

Avaliar o perfil e as preferências dos cirurgiões de catarata que atuam na Região Sul do Brasil por meio de um questionário voluntário e confidencial. 


\section{MÉTODOS}

Procurou-se elaborar um questionário (tabela 1) que permitisse, primeiramente, definir o perfil do cirurgião sem identificá-lo pessoalmente, buscando informações como: idade, sexo, tempo de profissão, modalidade de atendimento (público ou privado) e volume cirúrgico. As demais perguntas visavam identificar as preferências relativas à rotina operatória de cada um, incluindo método de esterilização, ambiente cirúrgico, tipo de anestesia, medicamentos, soluções e tipo de lente intraocular implantada.

Junto a esse questionário, foram anexados um envelope pré-selado, a ser usado para envio da resposta, e uma carta de apresentação, onde se explicava o propósito da enquete. Esses itens foram enviados a 1.000 oftalmologistas cadastrados na mala direta do Serviço de Oftalmologia da Santa Casa de Porto Alegre, RS. Na análise dos dados recebidos, usou-se o pacote estatístico SPSS 8.0 for Windows ${ }^{\circledR}$, Chicago, Il, USA.

\section{RESULTADOS}

Foram recebidas 210 respostas, o que corresponde a $21 \%$ dos formulários enviados. Sete delas precisaram ser excluídas: três por não terem sido devidamente respondidas e quatro porque o oftalmologista em questão já não operava mais. A maioria das respostas veio do Estado do Rio Grande do Sul $(41,4 \%)$, seguida pelas do Paraná $(38,4 \%)$ e de Santa Catarina $(20,2 \%)$. A tabela 2 mostra a distribuição do número respostas por estado ( $n=203,7$ exclusões), bem como a distribuição por local de atuação profissional (capital e interior). A tabela 3 mostra o número de questionários enviados para cada estado.

A idade dos entrevistados varia entre 24 e 72 anos, com média de 42,8 anos. Deles, 87,6\% pertencem ao sexo masculino. O tempo médio de profissão é de 15,8 anos e a prática individual constitui a modalidade de trabalho mais comum $(58,2 \%)$.

Para a maioria $(80,8 \%)$, o número de cirurgias mensais é inferior a 15 , sendo boa parte desse volume $(62,6 \%)$ constituída por pacientes com convênios/seguros de saúde. Nem todos $(40,8 \%)$ referiram usar termo de consentimento pré-operatório.

Quanto à rotina pré-operatória (tabela 4), 18\% disseram realizar regularmente o exame de ecografia ocular. Número que se mostrou maior $(64,6 \%)$ nos casos em que a observação do fundo de olho é prejudicada pelo grau de opacificação do cristalino. A ecobiometria faz parte da rotina de $92 \%$ dos entrevistados, sendo que $81 \%$ deles costumam também realizá-la no olho contralateral, quando o comprimento axial do que será operado encontra-se fora da média.

O ambiente hospitalar foi o preferido pela maioria dos cirurgiões (68\%), os demais realizam suas cirurgias em clínicas privadas ou centros de cirurgia ambulatorial $(31,7 \%)$. Apenas $12,9 \%$ ainda recorrem à internação hospitalar do paciente. $\mathrm{Na}$ esterilização do material, 76,6\% utilizam a autoclave, os demais fazem uso de métodos os mais variados que vão desde soluções anti-sépticas, estufa, ultravioleta e óxido de etileno.
$\mathrm{Na}$ anestesia do paciente, $87,4 \%$ empregam o bloqueio peribulbar e $2 \%$, anestésico tópico. O restante divide-se entre o uso da anestesia geral, bloqueio retrobulbar e subtenoniano. A presença do anestesiologista na sala de cirurgia é adotada por $87,6 \%$ dos cirurgiões, dos quais apenas metade recorre à sedação. A tabela 5 mostra a relação entre o número de procedimentos por mês e a presença do anestesiologista na sala cirúrgica.

Em relação à técnica cirúrgica, a extração extracapsular constitui a preferência de $89,7 \%$ dos entrevistados, sendo que $36,9 \%$ deles utilizam apenas esse método. Em contrapartida, $58,6 \%$ referem empregar a facoemulsificação, embora apenas $9,4 \%$ o façam em todos os casos. Boa parte $(38,9 \%)$ costuma usá-la em $60 \%$ das suas operações. A técnica intracapsular ainda faz parte do arsenal de alguns entrevistados (4,5\%) para casos específicos ( 1 a $20 \%$ ), embora dois profissionais tenham afirmado realizá-la em todas as cirurgias.

Nos demais tempos cirúrgicos, os recursos mais utilizados são: incisão límbica $(54,6 \%)$, capsulorrexis $(58,1 \%$, sendo que $63 \%$ com cistítomo) e sutura com pontos separados $(81,4 \%)$. Apenas $17,6 \%$ dos entrevistados não utilizam sutura rotineiramente.

No que se refere à lente intra-ocular, 33,5\% dos entrevistados usam, preferencialmente, modelos de $7 \mathrm{~mm}, 22,2 \%$ de $6,5 \mathrm{~mm}$ e $15,2 \%$ de $5,25 \mathrm{~mm}$. Lentes dobráveis são apenas implantadas por $24,1 \%$ dos cirurgiões e multifocais, por $0,5 \%$. A implantação secundária é realizada por $76,6 \%$ dos entrevistados, dos quais $38,5 \%$ utilizam lentes de câmara posterior suturadas, 34,4\%, lentes de câmara anterior e 3,6\%, lentes de câmara posterior suturadas à esclera.

A preferência quanto às soluções usadas durante a cirurgia é mostrada na tabela 7. Várias opções foram apresentadas em relação aos medicamentos empregados no período pósoperatório, no entanto, foi possível perceber uma predominância $(95,1 \%)$ das associações de antibiótico e corticosteróide (gráfico 1 ).

\section{DISCUSS $\tilde{A} O$}

A idéia, aparentemente simples, de se colher informações através de um questionário, foi aqui prejudicada pelo reduzido número de respostas obtidas $(21 \%)$. Número esse, menor do que se costuma observar em trabalhos do mesmo tipo ${ }^{(3-4)}$.

De qualquer modo, as preferências registradas nesse estudo definem algumas tendências importantes. A solicitação mais freqüente de certos exames, por exemplo, indica haver maior precaução quanto à correta indicação da cirurgia (microscopia especular, teste de sensibilidade ao contraste e ecografia) e maior preocupação com a qualidade do resultado visual que irá ser alcançado (teste de função macular e ecobiometria bilateral) ${ }^{(5-6)}$.

Também significativo é o número relativamente baixo de cirurgias realizadas mensalmente pela maior parte desses médicos. Isso mostra que o volume cirúrgico nessa região do País não é proporcional ao número de portadores de catarata. Se- 


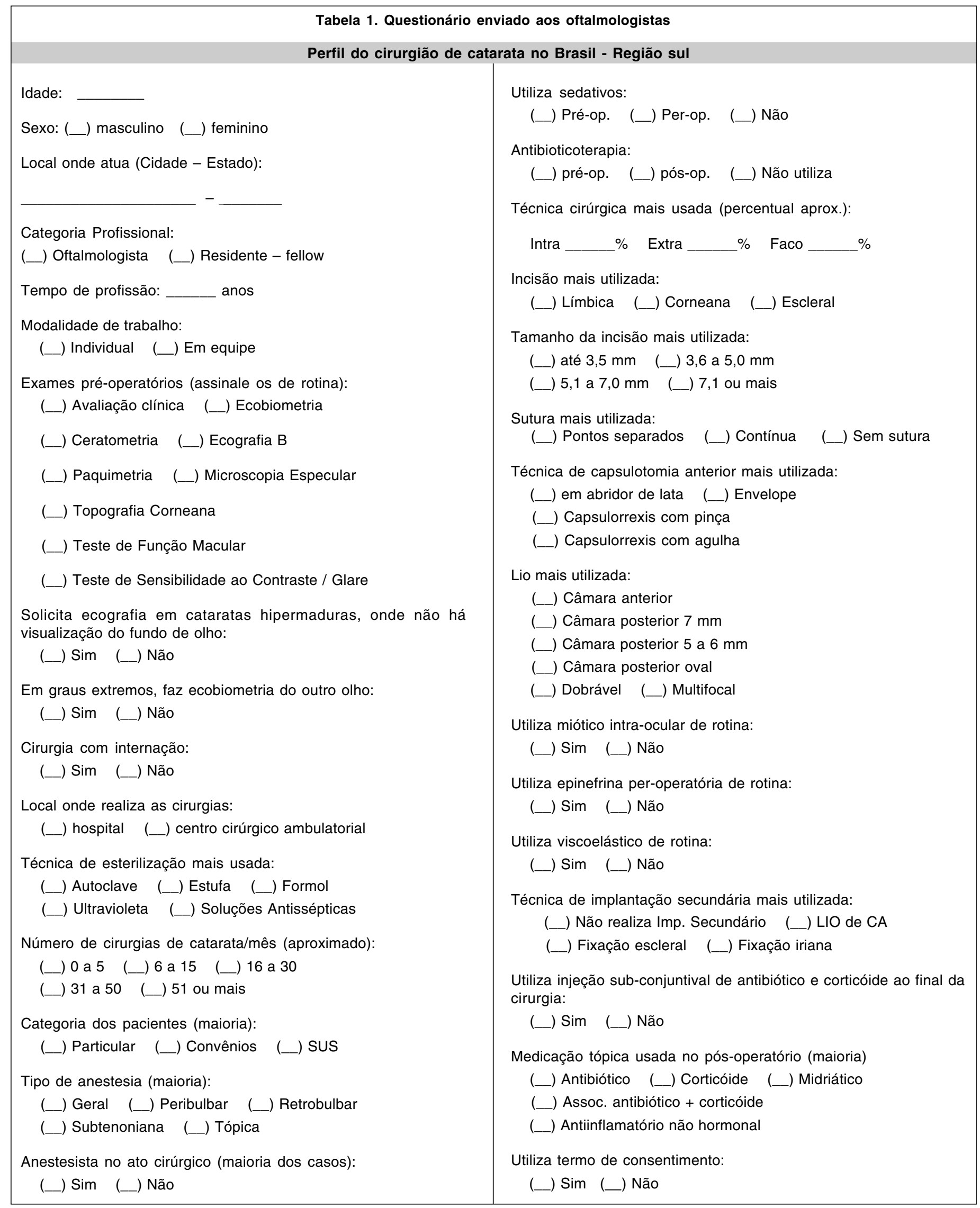




\begin{tabular}{|c|c|c|c|}
\hline \multicolumn{4}{|c|}{ Tabela 2. Respostas por estado - capital $x$ interior } \\
\hline Estado & Capital & Interior & Total no Estado \\
\hline Rio Grande do Sul & $38(45,2 \%)$ & $46(54,8 \%)$ & $84 \quad(41,4 \%)$ \\
\hline Santa Catarina & $6(14,6 \%)$ & $35(85,4 \%)$ & $41 \quad(20,2 \%)$ \\
\hline Paraná & $27(34,6 \%)$ & $51(65,4 \%)$ & $78 \quad(38,4 \%)$ \\
\hline Total & $71(35,0 \%)$ & $132(65,0 \%)$ & $203(100,0 \%)$ \\
\hline
\end{tabular}

\begin{tabular}{|c|c|}
\hline \multicolumn{2}{|c|}{ Tabela 3. Questionários enviados por estado } \\
\hline Estado & Número de questionários \\
\hline Rio Grande do Sul & $433(43,3 \%)$ \\
\hline Santa Catarina & $196(19,6 \%)$ \\
\hline Paraná & $371(37,1 \%)$ \\
\hline Total & $1000(100,0 \%)$ \\
\hline
\end{tabular}

\begin{tabular}{|llr|}
\hline \multicolumn{3}{|c|}{ Tabela 4. Exames pré-operatórios de rotina } \\
Avaliação Clínica & $(n=194 / 202)$ & $96,0 \%$ \\
Ecobiometria & $(n=186)$ & $92,1 \%$ \\
Ceratometria & $(n=162)$ & $80,2 \%$ \\
Teste de função macular & $(n=68)$ & $33,7 \%$ \\
Microscopia especular & $(n=37)$ & $18,3 \%$ \\
Ecografia B & $(n=36)$ & $17,8 \%$ \\
Topografia corneana & $(n=27)$ & $13,4 \%$ \\
Teste de sensibilidade ao contraste/glare & $(n=18)$ & $8,9 \%$ \\
Paquimetria & $(n=16)$ & $7,9 \%$ \\
Relação de exames complementares realizados no pré-operatório de catarata \\
e a freqüência de entrevistados que os solicitam como rotina pré-operatória \\
\hline
\end{tabular}

\begin{tabular}{|cccc|}
\hline \multicolumn{4}{|c|}{ Tabela 5. Anestesista na cirurgia x cirurgias/mês } \\
Anestesista na sala & \multicolumn{3}{c|}{} \\
$N^{\circ}$ de cirurgias/mês & Sim & Não & Não respondeu \\
1 a 5 & 63 & 12 & 2 \\
6 a 15 & 79 & 8 & - \\
16 a 30 & 22 & 4 & - \\
31 a 50 & 9 & 1 & - \\
51 ou mais & 3 & - & - \\
Relação entre o número de cirurgias/mês realizadas pelos entrevistados e o \\
acompanhamento anestesiológico durante a cirurgia \\
\hline
\end{tabular}

\begin{tabular}{|lc|}
\hline \multicolumn{2}{|c|}{ Tabela 6. Tempo cirúrgico: preferências } \\
Incisão límbica & $54,6 \%$ \\
Incisão escleral & $33,1 \%$ \\
Incisão corneana & $12,3 \%$ \\
Capsulorrexis & $58,1 \%$ \\
Capsulotomia em abridor de lata & $41,9 \%$ \\
Sutura com pontos separados & $81,4 \%$ \\
Não utiliza sutura & $17,6 \%$ \\
Pontos contínuos & $1,0 \%$ \\
\hline
\end{tabular}

\begin{tabular}{|lll|}
\hline \multicolumn{3}{|c|}{ Tabela 7. Produtos de uso trans-operatório } \\
Viscoelástico & $(n=192 / 202)$ & $95,0 \%$ \\
Antibiótico e corticóide subconjuntival & $(n=171 / 202)$ & $84,7 \%$ \\
Povidona tópica & $(n=159 / 201)$ & $79,1 \%$ \\
Miótico intra-ocular & $(n=108 / 202)$ & $53,5 \%$ \\
Epinefrina intra-ocular & $(n=56 / 199)$ & $28,1 \%$ \\
Medicamentos usados rotineiramente pelos oftalmologistas entrevistados em & \\
\hline suas cirurgias de catarata & & \\
\hline
\end{tabular}

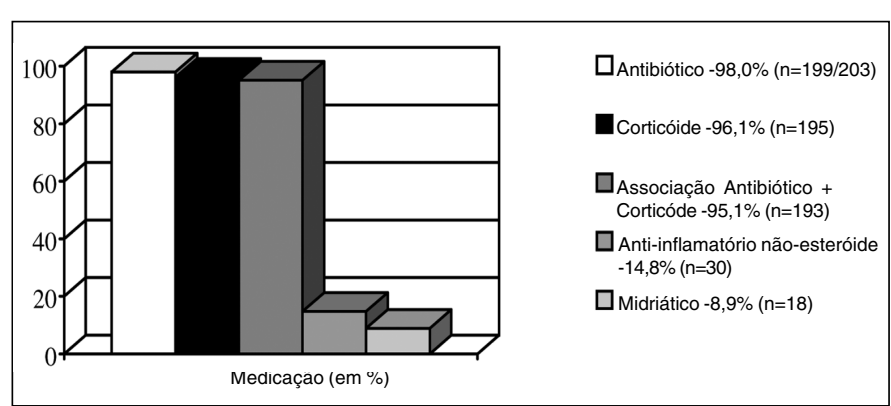

Gráfico 1 - Medicações tópicas rotineiramente utilizadas pelos oftalmologistas entrevistados no pós-operatório de suas cirurgias de catarata

gundo levantamentos do início da década de 90, estimava-se haver cerca de 350 mil cegos por catarata no Brasil ${ }^{(7-8)}$. Levantamentos do mesmo período calculavam que, para atender às demandas populacionais, seriam necessárias 450 mil cirurgias ao ano em nosso país. Este é um sinal de que esta patologia continua a ser uma importante causa tratável de cegueira ${ }^{(8)}$.

Observou-se também que os pacientes não estão sendo submetidos à internação hospitalar para a cirurgia, e que o procedimento ambulatorial está sendo adotado com o objetivo de otimizar o funcionamento da Sala de Cirurgia e minimizar o trauma emocional da cirurgia para o paciente. Tal fato, certamente está relacionado com as modernas técnicas de anestesia que permitem uma rápida recuperação do paciente e, por que não dizer, pela necessidade de redução de custos, imposta pela atual situação econômica mundial ${ }^{(9)}$.

Apesar disso, observa-se que a maioria dos oftalmologistas ainda prefere contar com a assistência do anestesista. Conscientes, sem dúvida, da gravidade das complicações anestésicas que, mesmo com toda a evolução e simplificação dos últimas décadas, ainda podem ocorrer ${ }^{(10-11)}$. De acordo com os dados colhidos neste estudo, nota-se que a presença do anestesista é mais comum entre os que possuem maior volume cirúrgico. A razão é bem simples: ao dividir as responsabilidades na sala de cirurgia, o cirurgião passa a ter maior tranqüilidade para desempenhar sua função primária.

Em relação à técnica cirúrgica, vê-se que, apesar de ter havido um aumento na quantidade de cirurgiões que utilizam a facoemulsificação - de 2,6\% em $1996^{(3)}$ para 39\% em 2002 - esse número ainda é muito pequeno, quando comparado ao de outros países, onde cerca de $80 \%$ das operações são feitas através dessa técnica ${ }^{(4-6)}$. 


\section{CONCLUSÃO}

Os dados obtidos nesse estudo sugerem que os avanços alcançados em relação à cirurgia de catarata (facoemulsificação) não têm sido plenamente empregados pelos oftalmologistas da região Sul do Brasil e, conseqüentemente, usufruídos por seus pacientes.

\section{ABSTRACT}

Purpose: To evaluate the profile and preferences of cataract surgeons working in southern Brazil. Methods: A questionnaire about the routines of cataract surgery was elaborated by the authors and sent to 1,000 ophthalmologists of the three states of southern Brazil (Paraná, Santa Catarina and Rio Grande do Sul). A presentation letter signed by the authors and a pre-sealed envelope were attached to the questionnaire. Data analysis was done using the statistical package SPSS 8.0 for Windows ${ }^{\circledR}$. Results: Only 210 completely filled out questionnaires were sent back by the interviewees. Through the analysis of the gathered data, one could observe certain preferences of these surgeons such as the use of peribulbar anesthesia and extracapsular cataract extraction with 5 to $6 \mathrm{~mm}$ opticplate-IOL implants and single stitch suture. Phacoemulsification is only used by $39 \%$ of these surgeons in about $60 \%$ of their cases. Conclusions: Regardless the constant technical developments seen in this area of medicine, it is clear that modern techniques employing phacoemulsification still are not used routinely by ophthalmologists in southern Brazil.

Keywords: Cataract; Cataract extraction; Phacoemulsification; Professional practice location; Physicians; Physician's practice patterns/statistics \& numerical data

\section{REFERÊNCIAS}

1. Kohnen T, Koch MJ. Refractive aspects of cataract surgery. Curr Opin Ophthalmol 1998;9:55-9.

2. Jampel RS. The effect of technology on the indications for cataract surgery. Doc Ophthalmol 1999;98:95-103.

3. Siqueira JM, Trindade FC. O perfil do cirurgião de catarata no Brasil 1996. Rev Bras Oftal 1996;55:855-60.

4. Leaming DV. Practice styles and preferences of ASCRS members - 1999 survey. J Cataract Refract Surg 2000;26:913-21.

5. Oshika T, Araie M, Masuda K, Majima Y, Leaming DV. Current trends in cataract and refractive surgery in Japan: 1996 survey. Jpn J Ophthalmol 1998; 42:227-41.

6. Krootila K. Practice and preferences of Finnish cataract surgeons - 1998 survey. Acta Ophthalmol Scand 1999;77:544-7.

7. Kara José N, Delgado AM, Arieta CE. Catarata. In: XI Congresso Brasileiro de Prevenção da Cegueira.; 1994; Brasília. Anais. 1994. p.17-8.

8. Kara José N, Alves MR. Catarata: Um problema de saúde pública. In: KaraJosé N. organizador. Prevenção da cegueira por catarata. Campinas: UNICAMP; 1996. p.11-8

9. Rosenfeld SI, Litinsky SM, Snyder DA, Plosker H, Astrove AW, Schiffman J. Effectiveness of monitored anesthesia care in cataract surgery. Ophthalmology 1999;106:1256-60; discussion p.1261.

10. To EW, Chan DT. Arteriovenous fistula induced by a peribulbar nerve block. J Cataract Refract Surgery 2000;26:1253-5.

11. Ribeiro RM. Apnéia pós-bloqueio peribulbar em cirurgia de catarata: relato de caso e revisão da bibliografia. Arq Bras Oftalmol 2000;62:262-4.

\section{CONGRESSO INTERNACIONAL DA SOCIEDADE BRASILIENSE DE OFTALMOLOCIA}

\section{6 e 27 de março de 2004}

\section{BRASÍLIA - DF}

INFORMAÇÕES: Tel/Fax.: (61) 248-4030/8116-3304

com Dr. Hilton Medeiros ou

Tel/Fax.: (61) 248-4030/346-6971

com Ana Paula 